\title{
Confidence in value-based choice
}

\author{
Benedetto De Martino ${ }^{1-3,6}$, Stephen M Fleming ${ }^{2,4-6}$, Neil Garrett ${ }^{1}$ \& Raymond J Dolan ${ }^{2}$
}

Decisions are never perfect, with confidence in one's choices fluctuating over time. How subjective confidence and valuation of choice options interact at the level of brain and behavior is unknown. Using a dynamic model of the decision process, we show that confidence reflects the evolution of a decision variable over time, explaining the observed relation between confidence, value, accuracy and reaction time. As predicted by our dynamic model, we show that a functional magnetic resonance imaging signal in human ventromedial prefrontal cortex (vmPFC) reflects both value comparison and confidence in the value comparison process. Crucially, individuals varied in how they related confidence to accuracy, allowing us to show that this introspective ability is predicted by a measure of functional connectivity between vmPFC and rostrolateral prefrontal cortex. Our findings provide a mechanistic link between noise in value comparison and metacognitive awareness of choice, enabling us both to want and to express knowledge of what we want.

The subjective confidence we have in our decision-making, and that of others, has far-reaching consequences. For example, the recommendations of a financial advisor who expresses high confidence in a particular investment option will carry more weight than one who is ambivalent. An expression of doubt in or caution concerning a particular course of action can lead one to question or revisit a previous decision. Previous work has established that the vmPFC has a central role in computing the value of potential choice options ${ }^{1-5}$, with activity in this region reflecting the dynamic evolution of a value comparison ${ }^{6}$. However, this work has focused exclusively on the choice process, without considering the subject's level of confidence in the decision. Consequently, it is unknown how a process of value comparison, instantiated in vmPFC, relates to subjective confidence.

Previous studies have reported neural correlates of decision confidence in brain regions associated with a value representation. For example, firing rates in rat orbitofrontal cortex ${ }^{7}$ and functional magnetic resonance imaging ( $\mathrm{fMRI}$ ) signal in human $\mathrm{vmPFC}^{8}$ show graded changes as perceptual decisions become more difficult. However, as these studies delineate confidence in terms of factors governing choice, they are unable to tease apart the relationship between trial-to-trial subjective confidence and decision value. In contrast, the field of perceptual decision-making has noted that confidence can be measured independently of the choice process itself ${ }^{9,10}$, where it is conceptualized as reflecting a 'second-order' metacognitive evaluation. Critically, dissociating confidence from other features of the decision process requires acquisition of separate measures of choice and confidence ${ }^{11}$.

Here we implement such an approach to dissociate value and confidence during decision-making and to identify their respective neural substrates. We collected trial-by-trial estimates of decision confidence while healthy volunteers chose between pairs of snack items. We also measured the subjective value of each snack item by means of a standard incentive-compatible bidding procedure. This allowed us to dissociate confidence from value, and in so doing provide evidence that confidence reflects an assessment of choice accuracy.

To explore systematic relationships between confidence, accuracy, choice and reaction time, we modeled our data using a variant of a race model ${ }^{7,12}$ (one of a larger class of dynamic models of decisionmaking $\left.{ }^{13}\right)$. This model predicts that subjective confidence reflects the stochastic accumulation of evidence during the value comparison process. As is consistent with this prediction, we show that the same anatomical region in ventromedial prefrontal cortex (vmPFC) not only reflects a difference in value between available options, but also the confidence associated with a value comparison process. Finally, we show that individual differences in participants' abilities to relate confidence to decision performance is linked to increased functional connectivity between vmPFC and rostrolateral prefrontal cortex (RLPFC), a region previously shown to function in metacognitive appraisal ${ }^{14}$.

\section{RESULTS}

We scanned twenty hungry participants while they made choices between food items that they could consume later (Fig. 1a). After making each choice, participants reported the degree of confidence in their decision (choice confidence). Note that confidence, or certainty, in the present study is conceptually distinct from risk, in that each choice determined a known outcome. Confidence here reflects the degree of subjective certainty in having made the best choice, which equates to choosing the higher valued item. To establish value for individual items, we asked participants at the end of the scanning session to place a bid for each food item using a standard incentive-compatible procedure, the Becker-DeGroot-Marschak (BDM) mechanism ${ }^{15}$. $\mathrm{BDM}$ is widely used in behavioral economics and neuroeconomics to elicit nonstrategic reservation prices, also known as willingnessto-pay. In this phase subjects were required to state their maximum willingness-to-pay for each food item (see Online Methods). Several studies have shown that this mechanism reliably elicits goal values

${ }^{1}$ Psychology and Language Sciences, University College London, London, UK. ${ }^{2}$ Wellcome Trust Center for Neuroimaging, at University College London, UK. ${ }^{3}$ Division of the Humanities and Social Sciences, California Institute of Technology, Pasadena, California, USA. ${ }^{4}$ Center for Neural Science, New York University, New York, New York, USA. ${ }^{5}$ Department of Experimental Psychology, University of Oxford, Oxford, UK. ${ }^{6}$ These authors contributed equally to this work. Correspondence should be addressed to B.D.M. (b.martino@ucl.ac.uk) or S.M.F. (fleming.sm@gmail.com). 
Figure 1 Task and behavioral results. (a) fMRI task (top): subjects were presented with a choice between two snacks and were then required to choose $(2.5 \mathrm{~s})$ one item to consume at the end of the experiment. After each choice, subjects indicated their level of confidence in having made a correct decision (choice confidence). Post-scanning task (bottom): subjects were presented with each item individually and had to submit a bid to buy each item. After each bid, they were asked to rate their level of confidence in having provided a correct bid price (bid confidence). (b) Probability of choosing the item on the right as a function of DV (that is, bid price) between the two items (logistic fit) for an exemplar subject (see Supplementary Fig. 2 for all individual subjects). Dashed line, all choices; black line, low-confidence choices; gray line, high-confidence choices. The red double-headed arrow indicates the increase in choice accuracy (change in slope) for high-versus low-confidence trials used in the between-subjects analyses (Figs. 4b and 5b). (c) The slope of the logistic fit is systematically higher (sharper) in highconfidence compared to low-confidence trials ( ${ }^{*} P<0.005 ; * * * P<0.0001$ ). (d) Average RT as a function of confidence and IDVI. (e) Heat map showing mean z-scored confidence (color bar) across subjects, as a function of subject-specific IDVI and RT quantiles. Error bars represent s.e.m. a
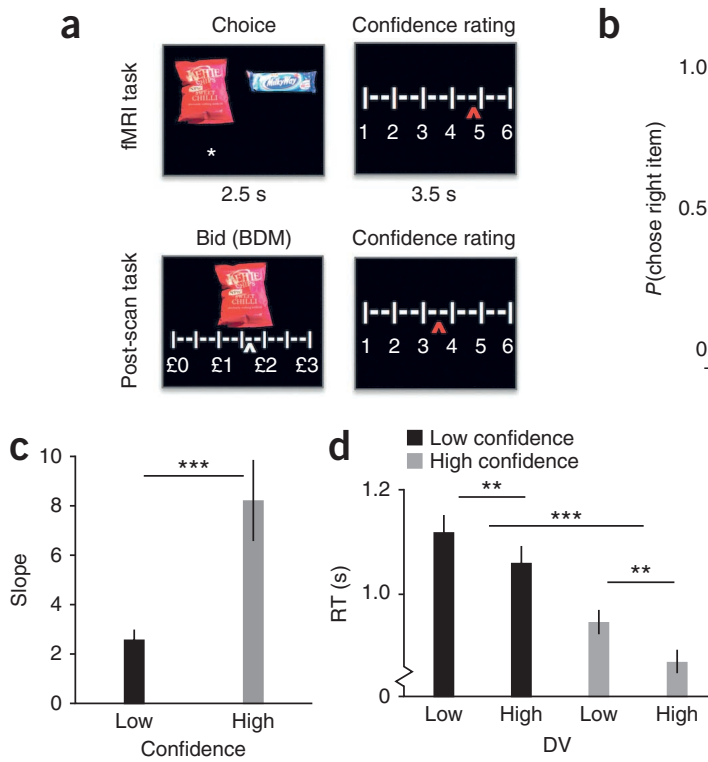

DV b

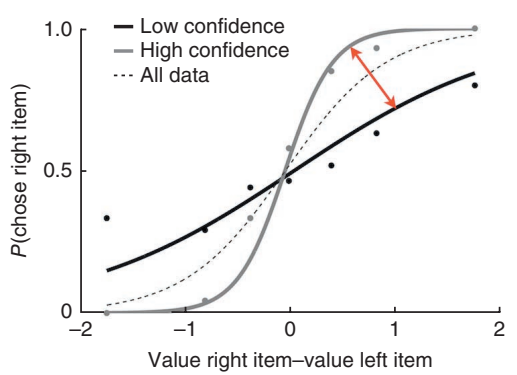

e

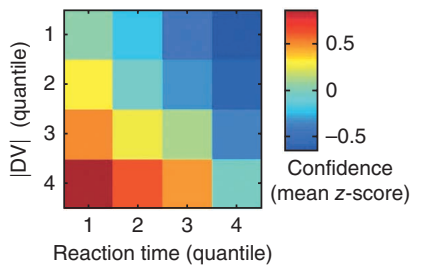

that are used by the decision maker to guide choice ${ }^{16-18}$. Participants also provided a rating of their confidence in each bid (bid confidence). Participants' bids for the leftmost items were then subtracted from bids for the rightmost items to calculate a signed difference in value (DV) between each pair of items, which was then entered into a logistic regression to predict the probability that the subject chose the rightmost item on each trial (Fig. 1b). In line with previous studies $^{2,19}$, we found that DV was a reliable predictor of participants' choices, with the slope of the logistic regression being a measure of choice accuracy, or noise in the choice process ${ }^{20}$.

\section{Choice, confidence and reaction time}

Unsigned $|\mathrm{DV}|$ only accounted for an average of $17.7 \%$ of the variance in participants' confidence ratings ( $r=0.42 \pm 0.19$, s.d.). This partial independence between confidence and $|\mathrm{DV}|$ allowed us to ask whether confidence reflects changes in choice accuracy (the selection of items with higher subjective value). By splitting our logistic regression fit into high- and low-confidence trials, we showed that higher confidence was consistently associated with increased choice accuracy (Fig. 1b,c and Supplementary Fig. 1). This effect of confidence on choice was also reflected in reaction time (RT), with main effects of both $|\mathrm{DV}|$ and confidence (both $P<0.001$ ) but no interaction (Fig. 1d). The three-way relationship between $|D V|$, confidence and RT is plotted in Figure 1e. We recognize that other factors (internal and external) besides $|\mathrm{DV}|$ and RT are likely to affect subjective confidence. We report a limited set of these factors (Supplementary Table 1) for which we could exercise good experimental control.

Using logistic regression, we next compared models of the interaction between confidence and value comparison. Choice confidence, unlike $\mathrm{DV}$, is in itself not a predictor of choice (right or left item) but instead refers to accuracy of the decision. We thus expected choice confidence to modulate the link between DV and choice. Model 1 predicted choice using DV alone; model 2 included choice confidence (that is, confidence at the decision time) as a modulator of DV (DV $\times$ confidence); models 3-5 examined whether bid confidence (that is, confidence at the bid time) could explain additional variance in the link between DV and choice (see Online Methods). In accordance with our predictions, model 2 provided a better account (that is, lower Bayesian information criterion (BIC)) of participants' choices than the other four models (Fig. 2a), as shown by the difference in BIC relative to model 2: model 1, 214.6; model 3, 196.2; model 4, 251.7; model 5, 111.9. Furthermore, model 2 was a better fit than the canonical model 1 in 19 of 20 participants as assessed by a likelihood ratio test $(\alpha=0.05)$. This analysis confirms that a critical modulator of choice accuracy is second-order confidence arising in the context of the comparison process (model 2) as opposed to first-order confidence in the item values (models 3-5).

\section{Stability of confidence over time}

We next examined whether the relationship between confidence and choice was stable over time. Splitting the logistic regression analysis into separate sessions revealed a robust main effect of confidence $\left(F_{1,19}=\right.$ 39.75; $P<0.0001)$ but a nonsignificant main effect of session $\left(F_{3,57}=\right.$ $0.3 ; P=0.7)$ and a lack of interaction between session and confidence $\left(F_{3,57}=0.13 ; P=0.9\right.$; Supplementary Fig. 2$)$. To examine whether local fluctuations in attention affected confidence, we constructed a serial autocorrelation regression model that predicted the current confidence rating from the confidence ratings given on the immediately preceding five trials, in addition to |DV|. None of the autocorrelation coefficients reached group-level significance (all $t<1.2, P>0.27$ ). Together these results indicate that confidence is a stable predictor of choice accuracy and that it does not reflect local changes in attention.

As each item pairing was presented twice (once in each spatial configuration), it was also possible to examine the relationship between confidence ratings given for identical choice pairs. As confidence is partly determined by absolute difference in value (|DV|, which does not vary across choice pairs), we expected some stability purely driven by DV. Thus, to address this question, we computed the partial correlation between first and second confidence ratings, controlling for DV. There was no significant difference between mean confidence ratings for the first and second presentations of the same item pairs $\left(t_{19}=-0.64, P=\right.$ 0.53). For 19 of 20 subjects, there was a significant partial correlation $(P<$ 0.05 ) between confidence ratings for repeated item pairs after controlling for the influence of $|\mathrm{DV}|$, indicating stability in confidence for judgments of particular item pairs that cannot be accounted for by $|\mathrm{DV}|$ alone. 
a

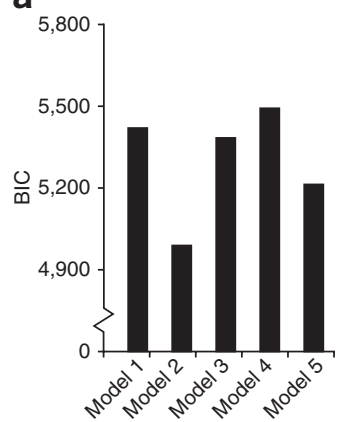

b

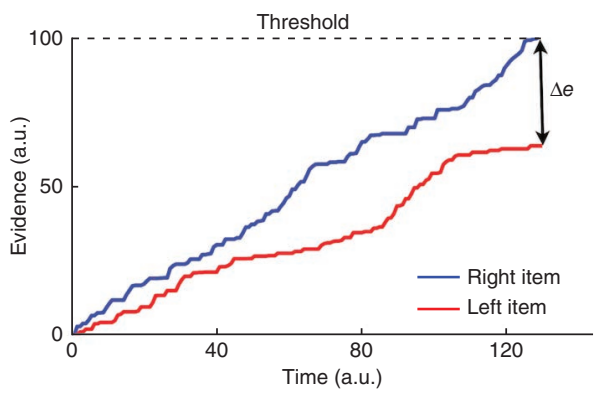

C

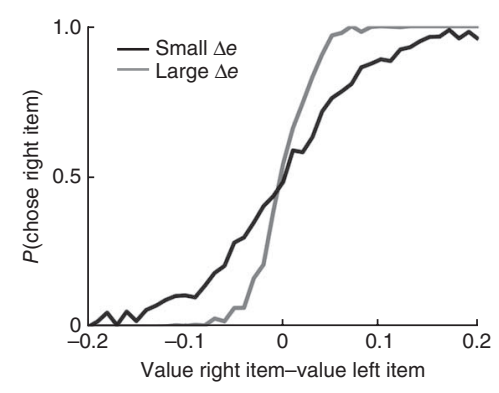

d

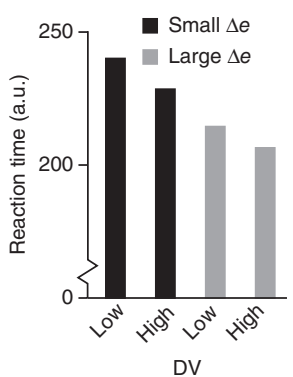

Figure 2 Computational model. (a) Comparison of regression models. Plotted are BIC scores (model 1: 5,424; model 2: 4,995; model 3: 5,388; model 4: 5,498; model 5: 5,291). Smaller numbers indicate a better model fit. See Results and Online Methods for details of each model. (b) Dynamic (race) model of value comparison for an example trial. Evidence in favor of each option accumulates over time, with a choice in favor of one or other option being made when threshold is reached. In this model, decision confidence is derived from the absolute difference between the two accumulators at the time of the decision $(\Delta e)$. a.u., arbitrary units. (c-e) Model predictions. (c) When $\Delta e$ is large (that is, high confidence) choice accuracy is predicted to increase, reflected by a sharper curve in the logistic regression. (d) RT values are predicted to decrease when either IDVI or $\Delta e$ increase. (e) Matrix representing how model confidence changes across IDVI and RT quantiles. Note the similarity between the model predictions and behavior (Fig. 1c-e).

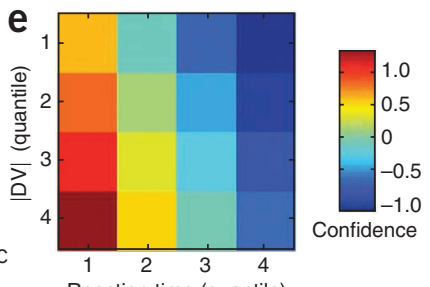

We then examined whether choices were stable over time. On average, $14.7 \%$ of choices ( $\pm 5.7 \%$ s.d.) were reversed on the second presentation. Choices that would be subsequently reversed were associated with significantly lower initial confidence than those that would subsequently be repeated (in arbitrary units: reversal confidence $=3.11$ \pm 0.72 (s.d.); repetition confidence $=4.40 \pm 0.54$ (s.d.); $t_{19}=12.1$, $\left.P<10^{-10}\right)$. In a logistic regression model predicting subsequent reversal from both $|\mathrm{DV}|$ and initial confidence, initial confidence was a significant negative predictor of choice reversal (mean standardized regression coefficient $-0.99 \pm 0.40$ (s.d.); one-sample $t$-test $\left.t_{19}=-11.2, P<10^{-9}\right)$. These data support a hypothesis that low confidence is associated with subsequent changes of mind.

\section{Race model}

Our best-fitting regression model suggested that confidence reflects accuracy in a value comparison. This led us to explore in more detail the precise mechanism by which confidence and value interact during the decision process. We adapted a race model ${ }^{12,21}$ wherein evidence in favor of each of the options (the snacks presented on the left and right sides of the screen) is accumulated over time and the decision is made on the basis of the first option to reach a threshold (Fig. 2b). In this model, confidence is defined as the absolute difference between the two accumulators at decision time $(\Delta e)$. Such a model predicts that when $\Delta e$ is large, then choice accuracy is increased, reflected by a sharper slope in the logistic regression (Fig. 2c). Thus, the race model neatly accounts for an increase in choice accuracy we observed behaviorally in the high-confidence condition (Fig. $\mathbf{1 b}$ and Supplementary Table 2). Furthermore this model predicts a decrease in RT when either $|\mathrm{DV}|$ or $\Delta e$ are increased (Fig. 2d), as seen in the behavioral data (Fig. 1d). The intuition is that, even within a particular level of initial DV, inter-trial noise in the value comparison process results in some trials having greater final DV values (higher confidence) than others. Such decisions will tend to be made more quickly, be more accurate and be associated with higher confidence (Fig. 2e). Indeed, this predicted relationship among RT, $|\mathrm{DV}|$ and confidence closely matched the behavioral data (Fig. 1e). Finally, since the model predicts that confidence reflects the stochastic evolution of a value comparison process, it will only be weakly related to initial DV. This feature of the model provides a parsimonious explanation for why DV and confidence are dissociable in our behavioral data.

\section{Confidence and value in vmPFC}

We next hypothesized that if choice confidence is an emergent property of a value comparison process, the same brain regions involved

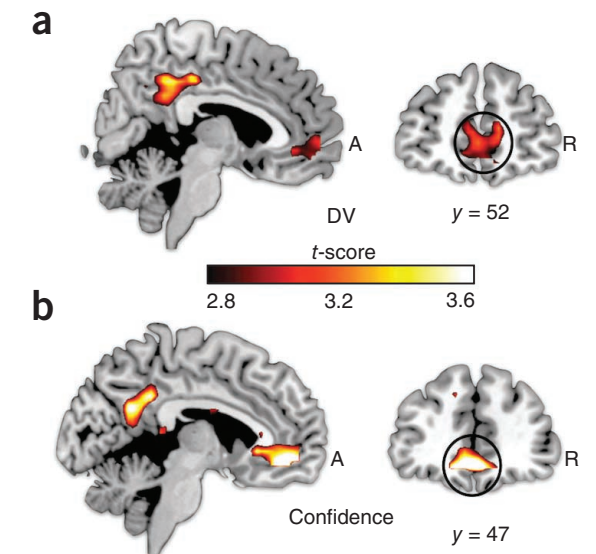

C

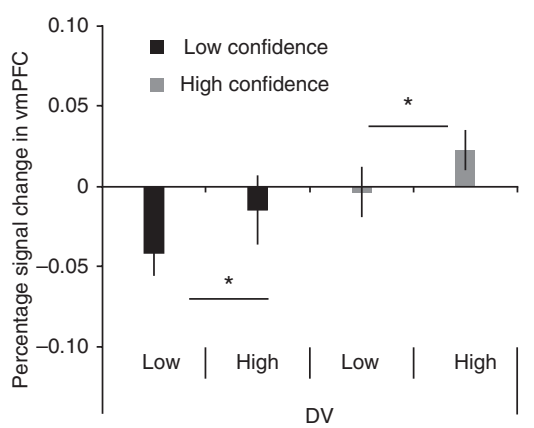

Figure 3 vmPFC. (a) Brain activity in precuneus and vmPFC. vmPFC (Montreal Neurological Institute (MNI) space coordinates $(x, y, z)=(12,56,4)$; circled) correlating with increases in DV between the two items presented $(P<0.05$, FWE corrected at cluster level). Left image, sagittal section; right image, coronal. (b) Brain activity in precuneus and vmPFC $(12,47,-11)$ correlating with increases in subjective confidence $(P<0.05 \mathrm{FWE}$ corrected at the cluster level). (c) Signal in vmPFC (6-mm sphere centered at the peak of the DV main effect $(12,56,4))$, showing main effects of DV and degree of confidence in the absence of an interaction. The plot (extracted from GLM 2; see Online Methods) is shown only to clarify the signal pattern in vmPFC (that is, lack of interaction between confidence and DV) and to confirm statistical inference (from GLM 1) regarding the main effects of DV and confidence. ${ }^{*} P<0.01$. Error bars represent s.e.m. 
a

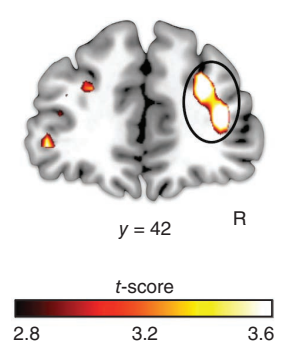

C

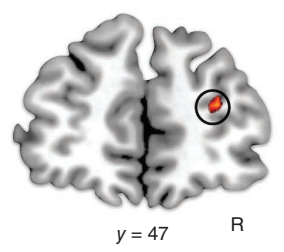

b
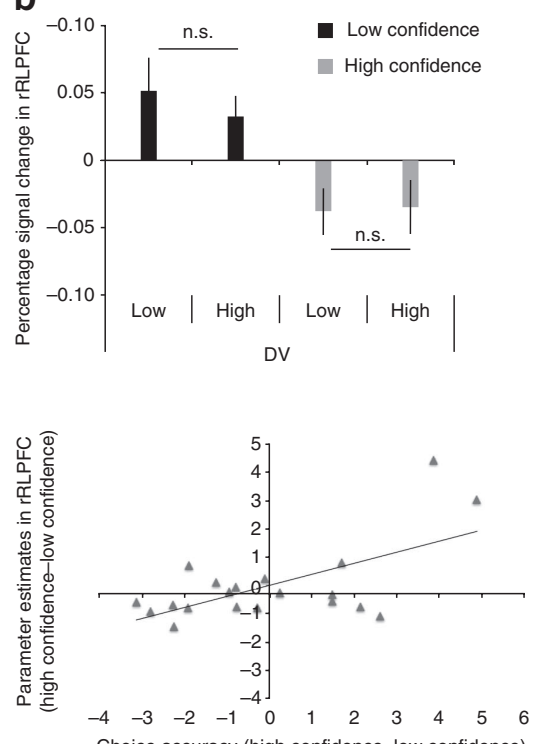

Choice accuracy (high confidence-low confidence)

$$
\triangle \text { Subjects }
$$

in value-based decision-making should also represent subjective confidence in a value estimate. In other words, if a brain region involved in value comparison is implementing a process akin to a race model ${ }^{6}$, then activity in that region should be modulated by both initial |DV| and noise (confidence) on that trial. To test this hypothesis, we constructed a general linear model (GLM) of our fMRI data in which each trial was modulated by two parametric regressors: $|D V|$ and confidence orthogonalized with respect to $|\mathrm{DV}|$. We show that activity in vmPFC was indeed modulated by both value and confidence (Fig. 3a,b and Supplementary Table 3; $P<0.05$ family-wise error (FWE) corrected at cluster level). This pattern is consistent with the established function of this region in encoding goal-values ${ }^{1,2}$ and with our hypothesis that this region also represents the confidence associated with a value comparison.

We next investigated whether $|\mathrm{DV}|$ and confidence interacted in vmPFC by splitting the model into high- and low-confidence trials, both parametrically modulated by $|\mathrm{DV}|$ (Fig. 2c). This analysis showed main effects

Figure 5 Connectivity analysis. (a) PPI analysis. Left: three-dimensional rendering, radiological orientation. Right: sagittal section. vmPFC (circled in black in right panel) shows increases in connectivity with a region of rRLPFC (6-mm sphere $(x, y, z)=(39,41,16)$; blue, left panel) previously identified as being modulated by confidence (vmPFC peak $(x, y, z)=(9,50,-11)$; R, right; $\mathrm{A}$, anterior. $P<0.05$, small-volume FWE corrected). $3 \mathrm{D}$ rendering made in mricron (radiological orientation). $\mathrm{R}$, right. $\mathrm{A}$, anterior. (b) Between-subjects regression analysis considering the increase in choice accuracy (see red doubleheaded arrow in Fig. 1b) between high-confidence and low-confidence conditions as a covariate for the modulation of connectivity (vmPFC peak $(x, y$, $z)=(15,56,-5) ; P<0.05$, small-volume FWE corrected). Coronal section. The scatter plot was not used for statistical inference (which was carried out in the SPM framework); it is shown solely for illustrative purposes. Error bars represent s.e.m.
Figure 4 RLPFC. (a) Brain activity in rRLPFC correlating with decreases in subjective confidence ( $P<0.005$, small-volume FWE corrected).

Coronal section; $\mathrm{R}$, right. (b) Signal in rRLPFC (6-mm sphere MNI space coordinates $(x, y, z)=(39,41,16))$ showing a main effect of confidence but not DV. The plot (extracted from GLM 2; see Online Methods) is shown only to clarify the signal pattern in rRLPFC (that is, absence of main effect of DV). n.s., not significant. (c) Between-subjects regression analysis considering the change in choice accuracy (slope of the logistic fit) between low- and high-confidence trials (see red double-headed arrow in Fig. 1b) as a covariate for confidence-related activity in rRLPFC (peak $(x, y, z)=(27$, 44, 16); $P<0.05$, small-volume FWE corrected). The scatter plot is not used for statistical inference (which was carried out in the SPM framework); it is shown solely for illustrative purposes. Error bars represent s.e.m.

of $|D V|$ and confidence in vmPFC but no interaction between them $(2 \times 2$ ANOVA with factors value, confidence: main effect of value $F_{1,19}=5.1, P<0.05$; main effect of confidence $F_{1,19}=7.6, P<0.05$; interaction $\left.F_{1,19}=0.7, P>0.5\right)$ (Fig. 3c). The absence of an interaction at the neural level is consistent with a theoretical independence between value and noise in the choice process, such that one can have high confidence in a low-value choice and vice versa. Furthermore, the pattern across conditions closely resembles that seen for RT values (Fig. 1d) providing convergent evidence that vmPFC activity is tightly linked to behavior. We also confirmed that the response to confidence was not driven by a categorical response to errors ${ }^{8}$ (Supplementary Fig. 3).

\section{Confidence in right rostrolateral prefrontal cortex}

A key question is how confidence-related information represented in vmPFC becomes available for self-report. One computationally plausible hypothesis is a hierarchical model wherein confidence in a comparison process is 'read out' by an anatomically distinct secondorder network ${ }^{22-24}$. Right rostrolateral prefrontal cortex (rRLPFC) is a likely candidate, as this region is implicated in metacognitive assessments of perceptual decisions $9,14,25$. Consequently, we tested whether this region acts more generally in metacognitive appraisal by enabling explicit report of confidence in a value comparison.

We first established that rRLPFC tracked changes in reported confidence but did not code for DV (Fig. 4a,b, Supplementary Fig. 4 and Supplementary Table 3; $P<0.005$, small-volume corrected (SVC)), as expected for a region providing a readout of decision confidence. 
Figure 6 Schematic of network relating confidence to subjective report. Summary of the relationship between our computational model and neuroimaging analyses. (a) Confidence in the decision $(\Delta e)$ emerges from the value comparison process instantiated in vmPFC. (b) To reach metacognitive awareness (and be reported by the participant), this information is transferred to $\mathrm{rRLPFC}$. The parameter $\sigma_{\text {conf }}$ governs the noise in the readout of $\Delta e$ (that is, decision confidence). If $\sigma_{\text {conf }}$ is zero, the information about confidence $(\Delta e)$ is uncorrupted, resulting in a pronounced shift in the choice accuracy between high-confidence and low-confidence trials (red double-headed arrows). As the level of metacognitive noise increases (higher values of $\sigma_{\text {conf }}$ ) the shift between the two curves (low and high confidence) diminishes. Differences in $\sigma_{\text {conf }}$ account for the inter-subject variability in metacognitive reportability we observed behaviorally. a.u., arbitrary units. a

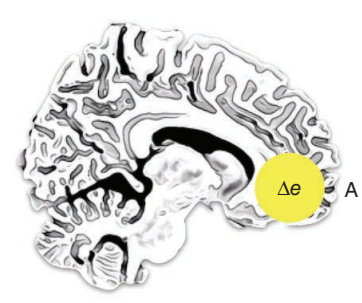

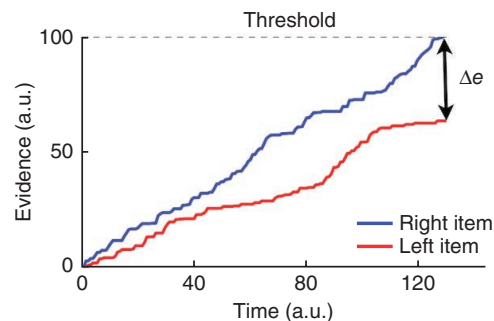

b

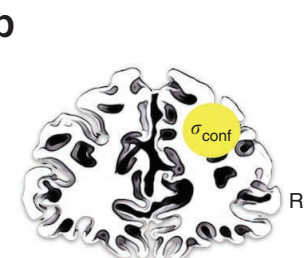

We next harnessed individual differences in metacognition to provide a more stringent test for the role of rRLPFC. We defined an individual's metacognitive accuracy as the change in choice accuracy (slope of the logistic fit) between low- and high-confidence trials (Fig. 1b). We reasoned that if rRLPFC acts in the metacognitive appraisal of confidence, activity in this region and/or its coupling with vmPFC should predict this change in slope across individuals. To test our first prediction, we entered change in slope as a between-subjects covariate in the whole-brain analysis of confidence-related activity, finding that this parameter significantly modulated the response to confidence in rRLPFC ( $P<0.05$; SVC for multiple comparisons). In other words, participants manifested a neurometric-psychometric match between their behavioral and neural responses to change in confidence level (Fig. 4c).

Metacognitive access: interaction between vmPFC and rRLPFC To test our second prediction, that these two regions are part of the same functional network (in the context of our task), we performed a psychophysiological interaction (PPI) analysis using rRLPFC as a seed (Fig. 5a). This analysis revealed a robust modulation of connectivity between $\mathrm{rRLPFC}$ and vmPFC $(P<0.05$ small-volume FWE corrected) by confidence level (Fig. 5a,b). Furthermore, the strength of connectivity between these two regions also predicted metacognitive accuracy across subjects (vmPFC; $P<0.05$; SVC for multiple comparisons) (Fig. 5b). Thus, both activity in rRLPFC itself and its coupling strength with vmPFC influenced the degree to which confidence was effectively read out for metacognitive report.

How might this readout process relate to our computational model of confidence? Intuitively, if reported confidence is a noisy facsimile of the confidence inherent in a decision process, the relationship between confidence and behavior will weaken and metacognitive accuracy will decrease ${ }^{26}$. We were able to modify the race model, introduced previously, to account for the inter-subject variability in metacognitive reports observed experimentally. We introduced an additional parameter $\left(\sigma_{\text {conf }}\right)$ governing the noise in the read-out of $\Delta e$ (that is, decision confidence) computed during the value comparison. Variation in this parameter captured variability in the change in slope between high- and low-confidence conditions, despite overall choice accuracy remaining equal (Fig. 6). Together with our imaging results, this analysis suggests that rRLPFC may indeed mediate variability in reported confidence (see Fig. 6 and Discussion).
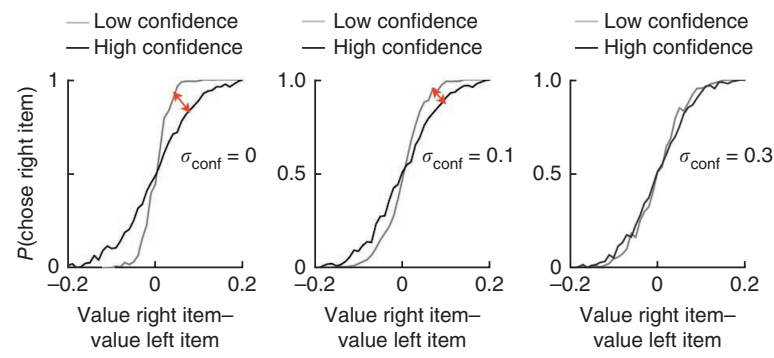

\section{DISCUSSION}

Here we show that decision confidence emerges from a value comparison process in vmPFC and that this region is in turn accessed by rRLPFC to enable a subjective assessment of confidence. Our neural findings are consistent with previous evidence showing that choice difficulty is coded by vmPFC in humans and analogous OFC neurons in rodents ${ }^{7,8}$. There is also an established body of work showing that this brain area represents the expected value of an outcome ${ }^{1-6}$. However, as previous studies defined confidence in terms of factors governing choice, they were unable to tease apart the relationship between value and confidence. Our results go beyond these studies by dissociating subjective confidence from DV. In so doing, we demonstrate that neural activity in the same anatomical region represents both variables, suggesting that confidence and DV are separate behavioral manifestations of the same underlying decision variable.

Choice confidence can be seen to emerge from the dynamics of noisy accumulators in the race model $7,12,21$, leading to dual effects of DV and RT on confidence ${ }^{27}$. The race model has previously been proposed to account for decision confidence in perceptual decisionmaking. In keeping with recent research efforts that have incorporated dynamic models into the field of economic decision-making ${ }^{28}$, we find that this model captures several features of the relationship between choice, RT and confidence in a value-based choice model. The separation between confidence and BDM values in the present study provides a new perspective on how an underlying decision variable can be fractionated into distinct behavioral components. Given that DV and confidence had independent effects on vmPFC activity, this result provides convergent support for the idea that vmPFC acts as a dynamic accumulator of choice values ${ }^{6}$. Our findings also accord with a theoretical Bayesian scheme in which uncertainty, or precision, is an inherent property of the neural code ${ }^{29-31}$.

A central problem for computational models of metacognition is how confidence information is read out for appraisal and communication to others. It has been proposed ${ }^{22,24}$ that such a computation can be achieved by a two-layer neural network architecture, in which the second-order network receives information about the performance of the first-order network and uses this information to generate reports of confidence. Our fMRI data can be interpreted in this framework 
and suggests that rRLPFC is a plausible locus for this second-order network. First, rRLPFC represented confidence but not DV, as predicted for a brain region that has access to information about confidence but is not directly involved in value comparison. Second, both confidence-related activity in rRLPFC and coupling between rRLPFC and vmPFC predicted the relationship between confidence and accuracy across individuals. This result can be explained if the coupling between vmPFC and rRLPFC reflects the fidelity with which reported confidence tracks the evolution of a putative accumulator process in vmPFC (Figs. 2b and 6). Notably, confidence-related activity in rRLPFC is also seen in perceptual decision-making ${ }^{14}$, together with a modulation of connectivity with visual cortex. This pattern of findings suggests that rRLPFC might have a domain-general role in metacognitive evaluation of decision-making, supporting the notion of a segregated neural process governing metacognitive access $22,24,26$.

An alternative interpretation of our data is that information about choice confidence is coded elsewhere, perhaps in parallel to the construction of choice values, and is then communicated to vmPFC (possibly via rRLPFC), where it is incorporated into the choice process. This mechanism would be analogous to a modulation of the vmPFC value signal during self-control by dorsolateral $\mathrm{PFC}^{32}$. Resolving this possibility is beyond the design of the current study and will require techniques with high temporal resolution, such as magnetoencephalography, that can track the evolution of confidence and valuation in the brain.

Our data show that humans have metacognitive access to noise in a value comparison and that increased choice accuracy is associated with high subjective confidence. In other words, although choices often appear noisy from the point of view of the experimenter ${ }^{20,33}$, subjective confidence ratings reveal systematic changes in this noise, reflected by changes in choice accuracy. Metacognitive access to confidence in a value comparison is likely to be useful for revisiting a choice that did not turn out as expected. Alternatively, but not mutually exclusively, metacognitive access may facilitate communication of confidence to others ${ }^{34}$, as when a financial advisor directs a client toward one stock option over another.

By integrating computational modeling with neural analysis, we provide evidence that subjective confidence is integral to the brain's representation of value in the vmPFC. Our work outlines a neural schema for how confidence-related information is computed and transferred to a distinct brain region (rRLPFC), supporting metacognitive report. Far from being a blind process of selection corrupted by noise, it would appear that value-based choices are accompanied by fluctuations in subjective confidence. A metacognitive access to value computation enables us not only to want but also to know what we want.

\section{METHODS}

Methods and any associated references are available in the online version of the paper.

Note: Supplementary information is available in the online version of the paper.

\section{ACKNOWLEDGMENTS}

We thank T. Fitzgerald, D. Kumaran and T. Sharot for comments on a previous draft of this manuscript, and T. Behrens and N. Daw for discussions. This work was supported by a Wellcome Trust Senior Investigator Award, 098362/Z/12/Z to R.J.D.; S.M.F. and B.D.M. are supported by Sir Henry Wellcome Fellowships (B.D.M., 082674/Z/07/Z; S.M.F., WT096185). The Wellcome Trust Centre for Neuroimaging is supported by core funding from the Wellcome Trust, 091593/Z/10/Z.

\section{AUTHOR CONTRIBUTIONS}

B.D.M. and S.M.F. conceived and designed the study. B.D.M., N.G. and S.M.F. developed stimuli and gathered and analyzed behavioral and fMRI data. B.D.M., S.M.F. and R.J.D. interpreted the data and wrote the paper.

\section{COMPETING FINANCIAL INTERESTS}

The authors declare no competing financial interests.

Published online at http://www.nature.com/doifinder/10.1038/nn.3279.

Reprints and permissions information is available online at http://www.nature.com/ reprints/index.html.

1. Rangel, A. \& Hare, T. Neural computations associated with goal-directed choice. Curr. Opin. Neurobiol. 20, 262-270 (2010).

2. FitzGerald, T.H.B., Seymour, B. \& Dolan, R.J. The role of human orbitofrontal cortex in value comparison for incommensurable objects. J. Neurosci. 29, 8388-8395 (2009).

3. Kable, J.W. \& Glimcher, P.W. The neural correlates of subjective value during intertemporal choice. Nat. Neurosci. 10, 1625-1633 (2007).

4. Basten, U., Biele, G., Heekeren, H.R. \& Fiebach, C.J. How the brain integrates costs and benefits during decision making. Proc. Natl. Acad. Sci. USA 107, 21767-21772 (2010)

5. Smith, D.V. et al. Distinct value signals in anterior and posterior ventromedial prefrontal cortex. J. Neurosci. 30, 2490-2495 (2010).

6. Hunt, L.T. et al. Mechanisms underlying cortical activity during value-guided choice. Nat. Neurosci. 15, 470-476 (2012).

7. Kepecs, A., Uchida, N., Zariwala, H.A. \& Mainen, Z.F. Neural correlates, computation and behavioural impact of decision confidence. Nature 455, 227-231 (2008).

8. Rolls, E.T., Grabenhorst, F. \& Deco, G. Choice, difficulty, and confidence in the brain. Neuroimage 53, 694-706 (2010).

9. Fleming, S.M., Weil, R.S., Nagy, Z., Dolan, R.J. \& Rees, G. Relating introspective accuracy to individual differences in brain structure. Science 329, 1541-1543 (2010).

10. Pleskac, T.J. \& Busemeyer, J.R. Two-stage dynamic signal detection: A theory of choice, decision time, and confidence. Psychol. Rev. 117, 864-901 (2010).

11. Kepecs, A. \& Mainen, Z. A computational framework for the study of confidence in humans and animals. Philos. Trans. R. Soc. Lond. B Biol. Sci. 367, 1322-1337 (2012).

12. Vickers, D. Decision Processes in Visual Perception (Academic Press, 1979).

13. Bogacz, R., Brown, E., Moehlis, J., Holmes, P. \& Cohen, J.D. The physics of optimal decision making: a formal analysis of models of performance in two-alternative forced-choice tasks. Psychol. Rev. 113, 700-765 (2006).

14. Fleming, S.M., Huijgen, J. \& Dolan, R.J. Prefrontal contributions to metacognition in perceptual decision making. J. Neurosci. 32, 6117-6125 (2012).

15. Becker, G.M., DeGroot, M.H. \& Marschak, J. Measuring utility by a single-response sequential method. Behav. Sci. 9, 226-232 (1964).

16. Plassmann, H., O'Doherty, J. \& Rangel, A. Orbitofrontal cortex encodes willingness to pay in everyday economic transactions. J. Neurosci. 27, 9984-9988 (2007)

17. De Martino, B., Kumaran, D., Holt, B. \& Dolan, R.J. The neurobiology of referencedependent value computation. J. Neurosci. 29, 3833-3842 (2009).

18. Hare, T.A., O'Doherty, J., Camerer, C.F., Schultz, W. \& Rangel, A. Dissociating the role of the orbitofrontal cortex and the striatum in the computation of goal values and prediction errors. J. Neurosci. 28, 5623-5630 (2008).

19. Boorman, E.D., Behrens, T.E.J., Woolrich, M.W. \& Rushworth, M.F.S. How green is the grass on the other side? Frontopolar cortex and the evidence in favor of alternative courses of action. Neuron 62, 733-743 (2009).

20. Sugrue, L.P., Corrado, G.S. \& Newsome, W.T. Choosing the greater of two goods: neural currencies for valuation and decision making. Nat. Rev. Neurosci. 6, 363-375 (2005).

21. Vickers, D. Evidence for an accumulator model of psychophysical discrimination. Ergonomics 13, 37-58 (1970).

22. Insabato, A., Pannunzi, M., Rolls, E.T. \& Deco, G. Confidence-related decision making. J. Neurophysiol. 104, 539-547 (2010).

23. Lau, H. \& Rosenthal, D. Empirical support for higher-order theories of conscious awareness. Trends Cogn. Sci. 15, 365-373 (2011).

24. Pasquali, A., Timmermans, B. \& Cleeremans, A. Know thyself: metacognitive networks and measures of consciousness. Cognition 117, 182-190 (2010).

25. Yokoyama, O. et al. Right frontopolar cortex activity correlates with reliability of retrospective rating of confidence in short-term recognition memory performance. Neurosci. Res. 68, 199-206 (2010).

26. Maniscalco, B. \& Lau, H. Comparing signal detection models of perceptual decision confidence. J. Vis. 10, 213 (2010).

27. Kiani, R. \& Shadlen, M.N. Representation of confidence associated with a decision by neurons in the parietal cortex. Science 324, 759-764 (2009).

28. Summerfield, C. \& Tsetsos, K. Building bridges between perceptual and economic decision-making: neural and computational mechanisms. Front. Neurosci. 6, 70 (2012).

29. Feldman, H. \& Friston, K.J. Attention, uncertainty, and free-energy. Front. Hum Neurosci. 4, 215 (2010).

30. Knill, D.C. \& Pouget, A. The Bayesian brain: the role of uncertainty in neural coding and computation. Trends Neurosci. 27, 712-719 (2004).

31. Friston, K. The free-energy principle: a unified brain theory? Nat. Rev. Neurosci. 11 127-138 (2010).

32. Hare, T.A., Camerer, C.F. \& Rangel, A. Self-control in decision-making involves modulation of the vmPFC valuation system. Science 324, 646-648 (2009).

33. Glimcher, P.W. Indeterminacy in brain and behavior. Annu. Rev. Psychol. 56, 25-56 (2005).

34. Bahrami, B. et al. Optimally interacting minds. Science 329, 1081-1085 (2010). 


\section{ONLINE METHODS}

Participants. Twenty-eight participants (mean age 24.24) took part in the study. Four participants were excluded because of excessive head motion. Three participants were excluded for erratic choice patterns that prevented reliable estimation of a logistic fit (an inverse temperature parameter five or more times larger than the average of the group). Participants were only included if they used a sufficient range of confidence ratings (s.d. $>0.8$ ) to allow estimation of metacognitive ability. This criterion led to the exclusion of one more subject. Twenty participants were included in the final analysis.

Scanning task. Participants were required to fast for $4 \mathrm{~h}$ before the study. During scanning they were required to make a series of binary choices between 19 common snack items $(2,000 \mathrm{~ms}$ ) to consume later (see Supplementary Table 4 for a list of items). Participants were asked to choose between each combination of items $(n=170)$ twice, counterbalanced across left-right spatial configurations (total number of choices $=340$ ) and divided into four sessions. After each choice, participants were asked to indicate their confidence in their decision (that is, "How confident are you that the choice you made was the right one for you?") on a continuous sliding scale between 1 (low confidence) and 6 (high confidence). Participants had 3,500 ms to move the pointer to the position that accurately reflected their confidence in the previous decision.

Post-scanning BDM task. Participants were presented each item on a computer screen and asked to submit a bid (from $£ 0$ to $£ 3$, using a sliding scale) to buy the item (unlimited time). After each bid, participants were asked to indicate their confidence in the bid they had just submitted (that is, "How confident are you that the bid you made was the right one for you?"; bid confidence) on a continuous sliding scale between 1 (low confidence) and 6 (high confidence). At the end of the experiment, one choice from the scanning phase was played out and the subject had the opportunity to buy the chosen item by means of an auction administered according to the Becker-DeGroot-Marschak (BDM) procedure ${ }^{15}$. More specifically, the experimenter randomly extracted a price from a uniform distribution ( $£ 0$ to $£ 3$ ) - the 'market price' of that item. If the participant's bidding price (willingness-to-pay) was above the market price, no transaction occurred. If the subject's bidding price was below the market price, the participant bought the snack item at the market price. At the end of the experiment, participants had to remain in the lab for an additional hour. During this hour, the only food they were allowed to consume was the item purchased in the auction, if any. This procedure encouraged subjects to choose preferred snacks during the scanning phase $\mathrm{e}^{16,18}$. Participants were compensated $£ 40$ for participation in the study. The price of any item purchased by a subject was deducted from this $£ 40$ participation fee.

Behavioral analysis and model. To examine the effect of value and confidence on choice we compared five candidate logistic regression models. All had the form

$$
P(c=R \mid X)=\Lambda(X \beta)
$$

where $\Lambda(x)$ is the logistic cumulative distribution function:

$$
\begin{gathered}
\Lambda(x ; u, s)=\frac{1}{1+e^{-\frac{x-u}{s}}} \\
P(c=R \mid X)=\frac{1}{1+e^{-a+b_{1} x_{1}+b_{2} x_{2}+\ldots b_{n} x_{n}}} \\
\log \frac{P(c=R)}{1-P(c=R)}=a+b_{1} x_{1}+b_{2} x_{2}+. . b_{n} x_{n}
\end{gathered}
$$

The simplest candidate model predicts the probability of choosing the rightmost option from the signed difference in value (DV), defined as $V_{\mathrm{R}}-V_{\mathrm{L}}$ :

$$
\text { Model 1: } X=\mathrm{DV}
$$

The slope of this function is assumed to result from randomness in choice ${ }^{20}$. If, on the other hand, subjects have metacognitive access to the noise in their decision process, we might expect choice confidence to modulate the impact of DV on choice:

$$
\text { Model 2: } X=\left[\mathrm{DV}\left(\mathrm{DV} \times \text { conf }_{\text {choice }}\right)\right]
$$

A second set of models examined whether confidence in the item price (bid confidence) modulates the link between DV and choice. On each trial there were two bid confidences (one for each item). Model 3 modulated DV by the mean bid confidence to enable direct comparison with model 2; model 4 split the DV predictor by bid confidence (low, high and mixed low/high, based on a subjectspecific median split); model 5 extended model 4 by including additional regressors for the modulation of choice confidence (that is, model 2 split by different bid confidences):

$$
\begin{aligned}
\text { Model 3:X }= & {\left[\mathrm{DV}\left(\mathrm{DV} \times \overline{\operatorname{conf}}_{\mathrm{BDM}}\right)\right] } \\
\text { Model 4: } X= & {\left[\mathrm{DV}_{\text {low }} \mathrm{DV}\right. \text { mixed }} \\
\text { Model 5: } X= & {\left[\mathrm{DV}_{\text {low }}\left(\mathrm{DV}_{\text {low }} \times \operatorname{conf}_{\text {choice }}\right)\right.} \\
& \mathrm{DV}_{\text {mixed }}\left(\mathrm{DV}_{\text {mixed }} \times \operatorname{conf}_{\text {choice }}\right) \\
& \left.\mathrm{DV} V_{\text {high }}\left(\mathrm{DV}_{\text {high }} \times \text { conf }_{\text {choice }}\right)\right]
\end{aligned}
$$

Models were compared via BIC scores using a fixed-effects analysis, where a difference in BIC of 5 indicates strong evidence for one model over another ${ }^{35}$. Two subjects were excluded from the analysis in models 4 and model 5 owing to a low variability in item confidence, precluding a median split. In addition, we assessed the improvement in model fit obtained for model 2 over nested model 1 for each subject individually using a likelihood ratio test $\left(\chi^{2}, 1\right.$ d.f.).

Dynamic model of value comparison (race model). To predict how value, confidence and reaction time interact during decision-making, we harnessed a dynamic model of the value comparison process ${ }^{7,21}$. In the race model, separate decision variables accumulate evidence for distinct options, with the final decision determined by which accumulator reaches threshold first. On each time step during accumulation, a new evidence sample is drawn from a normally distributed random variable $s_{t}=N\left(u_{\text {stim }}, \sigma_{\text {stim }}\right) \cdot u_{\text {stim }}$ is positive if the correct choice (higher value item) is the righthand item, negative if the correct choice is the left item. Because $s_{t}$ is drawn from a normal distribution, the actual value of $s_{t}$ at each time step may be positive or negative. The accumulators evolve according to the following equations:

$$
\begin{aligned}
R_{t+1} & =\left\{\begin{array}{l}
R_{t}+S_{t}, S_{t}>0 \\
R_{t}, S_{t} \leq 0
\end{array}\right. \\
L_{t+1} & =\left\{\begin{array}{l}
L_{t}, S_{t} \geq 0 \\
L_{t}-S_{t}, S_{t}<0
\end{array}\right.
\end{aligned}
$$

The race terminates when either $R_{t}$ or $L_{t}$ reach a predetermined threshold, $\theta$, with the decision being determined by which accumulator reaches threshold first. Therefore at decision time, $t(\theta)$, either $R_{t}$ or $L_{t}=\theta$. The finishing point of the losing accumulator depends on the values of $u_{\text {stim }}$ and $\sigma_{\text {stim. }}$.

An estimate of decision confidence, $\Delta e$, can be recovered from the race model as the distance between the two accumulators $R_{t}$ and $L_{t}$ at the time the race is terminated (Fig. 2b; refs. 7,12).

We simulated the model using the same parameters as in ref. 7. We simulated 1,000 trials at each level of $u_{\text {stim }}$ and recorded mean choice, confidence and reaction time. We display the simulation output in an identical manner to the behavioral data (Fig. 2c-e).

Even for identical levels of decision performance, it is known that the relationship between subjective confidence and decision-making varies between tasks and individuals ${ }^{9,36}$. We sought to account for this variability by introducing an additional parameter relating model confidence to subjective confidence, $\sigma_{\text {conf }}$. On each trial, reported confidence was drawn from a Gaussian distribution centered on $\Delta e$ : conf $=N\left(\Delta e, \sigma_{\text {conf }}\right)$. This feature of our model is consistent with the notion that reported confidence is derived from a higher-order stage of decision-making corrupted by noise ${ }^{26}$. We note that other functional forms for the link between model confidence and reported confidence are possible, but we do not investigate these here. We repeated the simulation three times with three levels of $\sigma_{\text {conf }}$, for a fixed $\sigma_{\text {stim }}$. Examination of psychometric function plots (Fig. 6b) shows that $\sigma_{\text {conf }}$ can account for the variability in change in slope observed across individuals. 
Image acquisition and analysis. Scanning acquisition was performed using a Siemens 3.0 T Allegra MRI Scanner (Erlangen, Germany). Gradient echo T2*weighted EPI (echoplanar) functional images with BOLD-sensitive contrast were acquired (imaging parameters: 48 transverse slices; TR, $2.88 \mathrm{~s}$; TE, $30 \mathrm{~ms} ; 3 \times$ 3 in-plane resolution; 2-mm slice thickness; 1 -mm gap between adjacent slices; $z$-shim $-0.4 \mathrm{mT} / \mathrm{m}$; positive phase encoding direction; slice tilt $-30^{\circ}$ ), optimized to detect changes in orbitofrontal cortex. For each subject, 228 volumes per session were collected (total number of volumes over four sessions $=912$ ), followed by a whole brain high-resolution T1-weighted anatomical structural scan and local field maps. Image analysis was performed using SPM8 (http://www.fil. ion.ucl.ac.uk/spm/). The first five volumes from each session were discarded to allow for T1 equilibration. Raw functional, structural and field map files were reconstructed using TBR. Field maps were reconstructed into a single phase file. This field map file was then used to realign and unwarp EPI functional images. Structural images were reregistered to mean EPI images and segmented into gray and white matter. These segmentation parameters were then used to normalize and bias correct the functional images. Normalized images were smoothed using a Gaussian kernel of $8 \mathrm{~mm}$ full-width at half-maximum.

General linear model 1 (parametric). Onset regressors beginning at the presentation of the two items were modulated by two parametric regressors: (i) unsigned difference in value $(|\mathrm{DV}|)$, defined as the absolute difference in value between the item presented on the right $\left(V_{R}\right)$ and the item presented on the left $\left(V_{L}\right)$, with values $V_{\mathrm{R}}$ and $V_{\mathrm{L}}$ ascertained from subjects' bids in the post-scanning phase (|DV| $\left.=\left|V_{R}-V_{L}\right|\right)$; and (ii) post-choice confidence ratings, which ranged from 0 to 500 on an arbitrary scale. In this model, confidence is orthogonalized with respect to |DV $\mid$ by the SPM8 software. Second, for each subject we constructed a separate GLM using a factorial design.

General linear model 2 (factorial). Events were split into regressors based on confidence level (that is, low and high confidence) using a median split for each individual subject. Each of these regressors was modulated by a $|\mathrm{DV}|$ parametric regressor (defined above).

Statistical inference. Second-level group contrasts from GLM 1 were calculated as one-sample $t$-tests against zero for each first-level linear contrast. Activations were reported as significant if they survived family-wise error correction (FWE) for multiple comparisons across the whole brain at the cluster level. For rRLPFC, we employed small-volume correction using an 8 - $\mathrm{mm}$ sphere centered on the coordinates $(36,44,28)$ taken from ref. 14. For GLM 2 , rfxplot ${ }^{37}$ (http://rfxplot. sourceforge.net/) was used to extract percentage signal change at each region of interest defined by $6-\mathrm{mm}$ spheres around the vmPFC and rRLPFC peak voxels from GLM 1. These values were entered into $2 \times 2$ ANOVAs (factors value, confidence) to further clarify the pattern of activity seen in GLM 1. In analysis of individual differences we employed small-volume correction (SVC) using 8 -mm spheres centered on the peak activations in vmPFC and rRLPFC taken from GLM 1.

Psychophysiological interaction analysis. To assess changes in connectivity between $\mathrm{rRLPFC}$ and vmPFC as a function of confidence, we carried out a psychophysiological interaction (PPI) analysis. PPI is a measure of context-dependent connectivity, explaining the regional activity of other brain regions (here, vmPFC) in terms of the interaction between responses in a seed region (here, rRLPFC) and a cognitive or sensory process. We used the second GLM (factorial) to run our PPI analysis (for details, see paragraph above). We carried out PPI analysis using the Generalised PPI toolbox for SPM (gPPI; http://www.nitrc.org/projects/ gppi/). gPPI creates a new GLM in which the deconvolved activity of the seed region is assigned to separate regressors dependent on the status of the original psychological variable (high or low confidence), and reconvolved with the hemodynamic response function. Average time courses were extracted from all voxels in a 6 - $\mathrm{mm}$ sphere surrounding the rRLPFC peak coordinate $(39,41,16)$. The main effects of high and low confidence, the seed-region time course and motion parameters were included as regressors of no interest. The PPI contrast compares high_conf ${ }^{\star}$ rRLPFC $(+1)$ with low_conf ${ }^{*}$ rRLPFC $(-1)$. This analysis showed a significant activation in $\operatorname{vmPFC}(9,50,-11)$ that reflects the increased connectivity between vmPFC and rRLPFC during high compared to low confidence trials.

35. Kass, R. Bayes factor. J. Am. Stat. Assoc. 430, 773-795 (1995).

36. Song, C. et al. Relating inter-individual differences in metacognitive performance on different perceptual tasks. Conscious. Cogn. 20, 1787-1792 (2011).

37. Gläscher, J. Visualization of group inference data in functional neuroimaging. Neuroinformatics 7, 73-82 (2009). 


\section{Supplementary Material}

\section{Confidence in value-based choice}

Benedetto De Martino, Stephen M. Fleming, Neil Garrett, Raymond J. Dolan 


\section{Supplementary Figures}

\section{Supplementary Figure 1 - Choice plots for all participants}
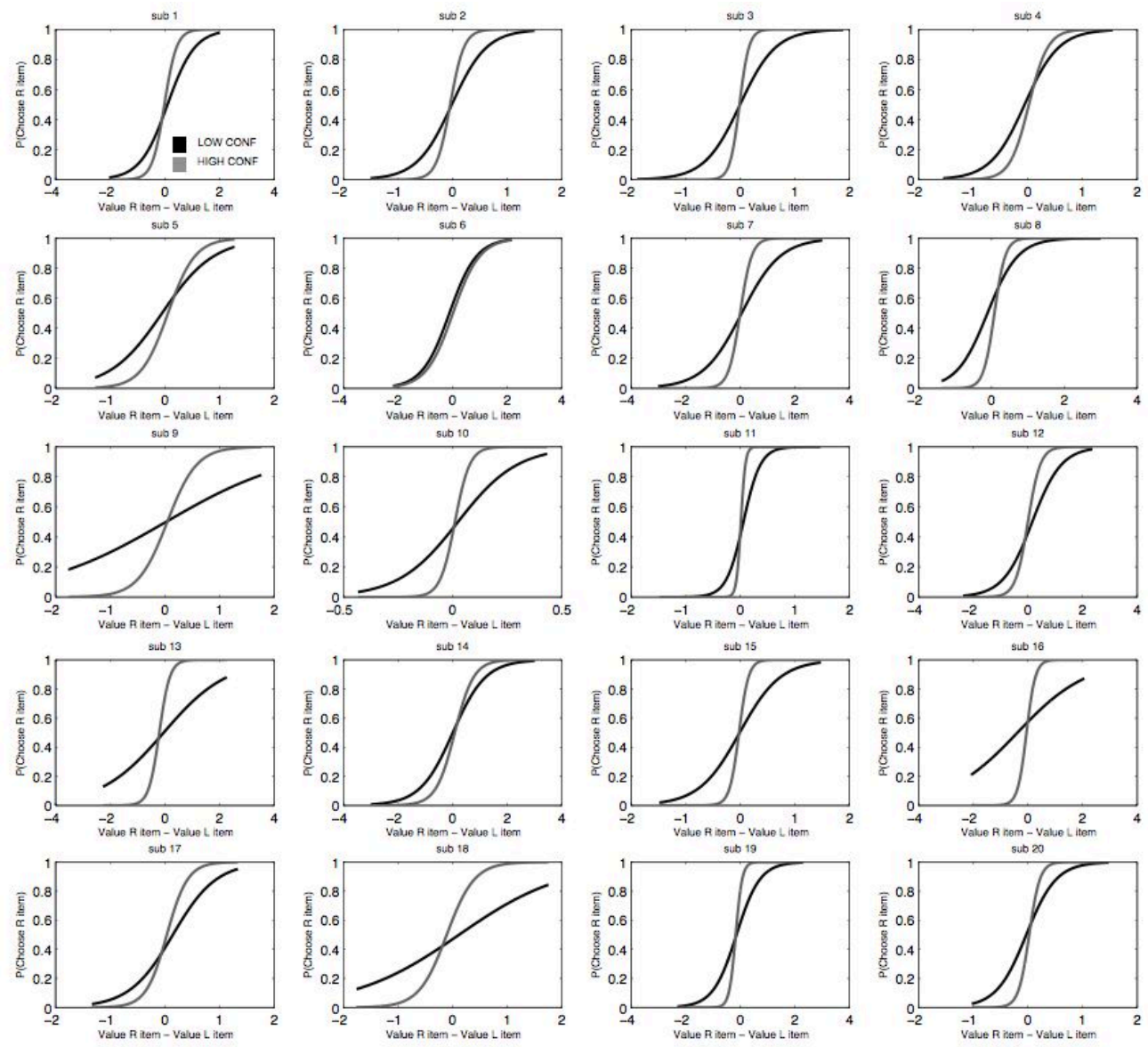

Probability of choosing the item on the right as a function of the difference in value (i.e. bid price) between the 2 items (logistic fit; black line = low confidence choices; grey line = high confidence choices). 


\section{Supplementary Figure 2 - Stability of the behavioural effect over time}

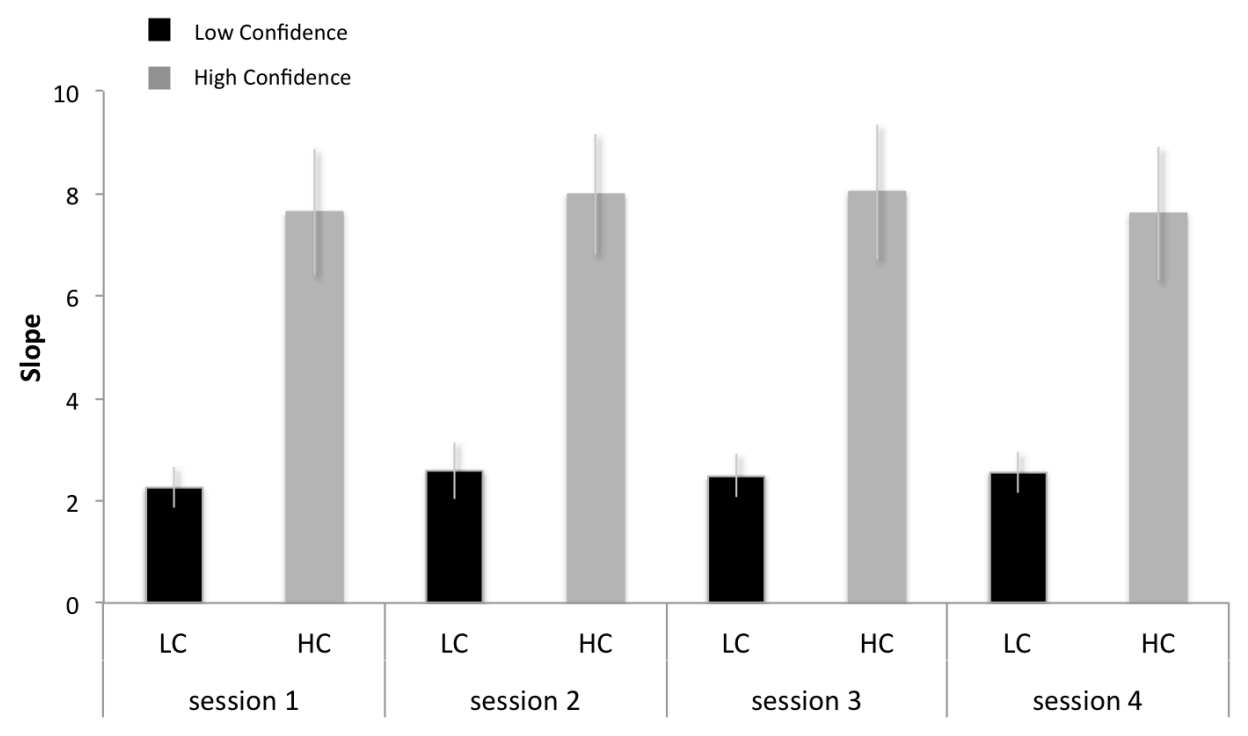

Slope from a logistic regression model predicting choice from DV, separately for high and low confidence and split by session.

Supplementary Figure 3 - Effect of correct/incorrect choice on the confidence signal in $v m P F C$
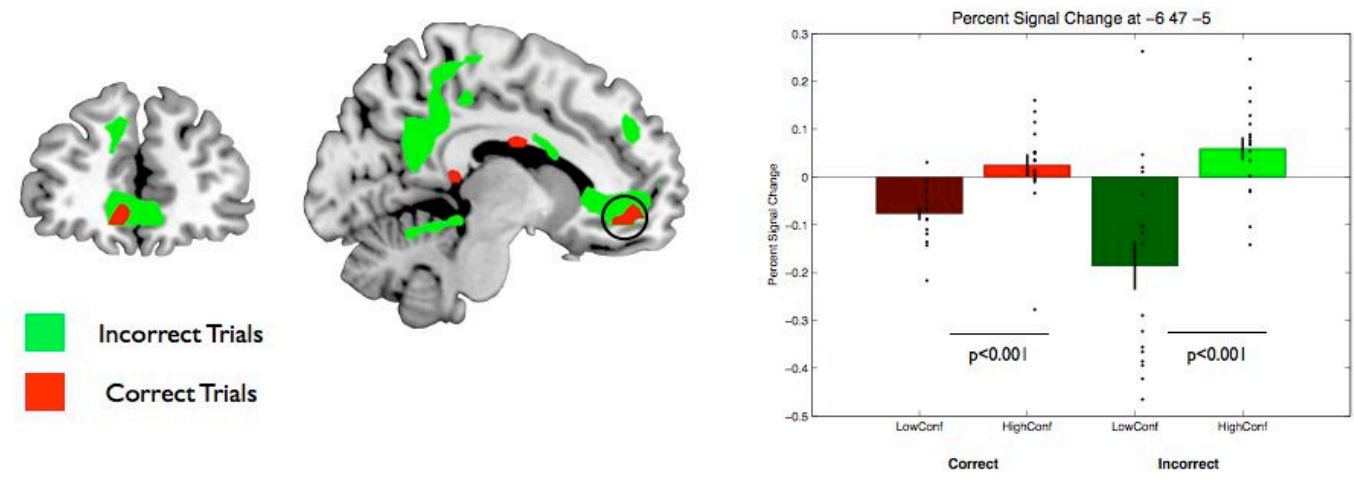

Here we ask whether the vmPFC response to confidence can be explained by a categorical difference between correct and error trials. We constructed another GLM similar to GLM 2 (see Methods) in which we split the categorical confidence regressor into two further regressors: one for correct trials (i.e. trials in which the participant chose the item with higher value - red in the figure) and one for incorrect trials (i.e. the participant chose the item with lower value - green in the figure). This analysis revealed a significant response in vmPFC to confidence in both conditions, confirming that the effect of confidence we identified was not driven by a categorical responses to errors. 


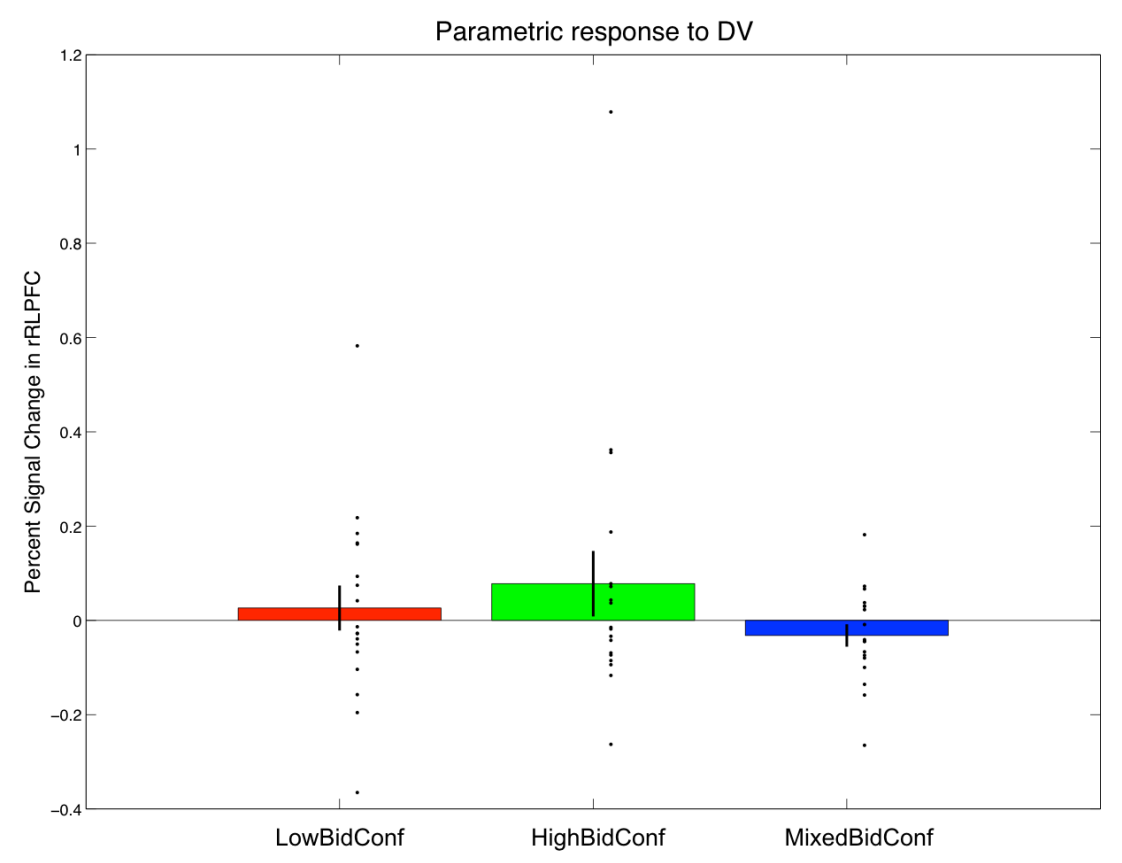

In this analysis each item was categorized (using a median split) into high or low bid confidence (using the bid confidence measured at the end of the experiment). This allowed us to construct a new parametric GLM (see Methods) in which each regressor is split into three new regressors: low bid confidence pairs, high bid confidence pairs, and mixed bid confidence pairs. Each regressor was parametrically modulated by both choice confidence and |DV|. Note that 2 subjects had to be excluded from this analysis due to a reduced variability in the bid confidence that did not allow a median split. This new model established that the activity of rRLPFC is insensitive to |DV| across all levels of bid confidence (in each of the 3 conditions the parametric response to |DV| in rRLPFC is not significantly different from zero -- one sample t-test $p>0.1$ ). This analysis excludes the possibility that a $|D V|$ signal in RRLPFC may have been masked by weak value signals in low bid confidence trials. The plot shows the parameter estimates for the LowBDMconf condition (green), the HighBDMconf condition (red) and the MixedBDMconf condition (blue). 


\section{Supplementary Tables}

Supplementary Table 1 - Analysis of additional factors affecting confidence and value

\begin{tabular}{|c|c|c|}
\hline Factor & Analysis & Results \\
\hline Item familiarity* & $\begin{array}{l}\text { Linear regression of } \\
\text { familiarity and }|D V| \text { on } \\
\text { confidence }\end{array}$ & $\begin{array}{l}\text { Mean familiarity was a } \\
\text { significant predictor of choice } \\
\text { confidence }\left(\mathrm{t}_{19}=3.09, \mathrm{P}<0.01 \text {, }\right. \\
\text { individually significant in } 13 \\
\text { out of } 20 \text { subjects); as } \\
\text { established previously, }|\mathrm{DV}| \\
\text { also significantly predicted } \\
\text { choice confidence in this } \\
\left.\text { model ( } \mathrm{t}_{19}=8.55, \mathrm{P}<0.001\right)\end{array}$ \\
\hline Actual retail price** & $\begin{array}{l}\text { Pearson correlation between } \\
\text { price and BDM bid }\end{array}$ & $\begin{array}{l}1 \text { out of the } 20 \text { participants } \\
\text { showed a significant positive } \\
\text { correlation between the } \\
\text { actual retail price of each } \\
\text { item and the bids they } \\
\text { submitted for these items } \\
\text { (group mean } r=0.0064 \pm \\
0.24 \text { ). }\end{array}$ \\
\hline Belief about retail price ${ }^{*}$ & $\begin{array}{l}\text { Pearson correlation between } \\
\text { price and BDM bid }\end{array}$ & $\begin{array}{l}4 \text { out of } 20 \text { subjects showed } \\
\text { a significant correlation } \\
\text { between their beliefs about } \\
\text { retail prices and the bids they } \\
\text { submitted for these items } \\
\text { (group mean } r=0.23 \pm \\
0.27 \text { ). }\end{array}$ \\
\hline Taste $^{\dagger}$ & $\begin{array}{l}\text { One-way repeated measures } \\
\text { ANOVA of mean confidence } \\
\text { by factor sweet/salty/mixed }\end{array}$ & $\begin{array}{l}\text { Confidence ratings were not } \\
\text { affected by item type }\left(F_{(1.273}\right. \\
24.182)=1.001, P=0.347)\end{array}$ \\
\hline Calorie content $^{\ddagger}$ & $\begin{array}{l}\text { One-way repeated measures } \\
\text { ANOVA of mean confidence } \\
\text { by factor high/low/mixed } \\
\text { calorie }\end{array}$ & $\begin{array}{l}\text { Confidence ratings were not } \\
\text { affected by calorie level } \\
\left(F_{(1.141,21.671)}=0.681, P\right. \\
=0.437)\end{array}$ \\
\hline
\end{tabular}

*Item familiarity and belief about retail price were collected in post-experiment questionnaires. ${ }^{* *}$ Actual retail prices were taken from a UK supermarket website. ${ }^{\dagger}$ Taste was determined by categorising each item as sweet or salty, and dividing post-choice confidence ratings into 3 groups: sweet (where both items in the choice pair comprised items categorised as sweet); salty (where both items in the choice pair comprised items categorised as salty); mixed (where a choice pair consisted of one item categorised as sweet and one item categorised as salty). ${ }^{\ddagger}$ Calorie content was determined by categorising items as high or low calorie (median split), and dividing post-choice confidence ratings into 3 groups: high calorie (where both items in the choice pair comprised items categorised as high calorie); low calorie (where both items in the choice pair comprised items categorised as low calorie); mixed calorie (where a choice pair consisted of one item categorised as high calorie and one item categorised as low calorie). 


\section{Supplementary Table 2 - Race model fits}

\begin{tabular}{|l|l|l|}
\hline Subject & $\boldsymbol{\sigma}_{\text {stim }}$ & $\boldsymbol{\sigma}_{\text {conf }}$ \\
\hline 1 & 1.33 & 0.22 \\
\hline 2 & 1.33 & 0.35 \\
\hline 3 & 1.13 & 0.11 \\
\hline 4 & 2.15 & 0.27 \\
\hline 5 & 1.54 & 0.14 \\
\hline 6 & 2.15 & 0.80 \\
\hline 7 & 1.33 & 0.11 \\
\hline 8 & 1.74 & 0.27 \\
\hline 9 & 2.36 & 0.27 \\
\hline 10 & 1.13 & 0.22 \\
\hline 11 & 1.13 & 0.11 \\
\hline 12 & 1.33 & 0.19 \\
\hline 13 & 1.74 & 0.16 \\
\hline 14 & 1.13 & 0.19 \\
\hline 15 & 0.72 & 0.16 \\
\hline 16 & $\mathrm{~N} / \mathrm{A}$ & $\mathrm{N} / \mathrm{A}$ \\
\hline 17 & 1.54 & 0.32 \\
\hline 18 & 1.74 & 0.47 \\
\hline 19 & 1.33 & 0.19 \\
\hline 20 & 1.33 & 0.24 \\
\hline Mean & 1.50 & 0.27 \\
\hline & & \\
\hline
\end{tabular}

Our race model simulations provide qualitative insight into the inter-relationship between confidence, DV and RT that we observe in the behavioural data (Fig. 1). Here we explore individual fits of model predictions to subject data. We held threshold constant, leaving two free parameters, $\boldsymbol{\sigma}_{\text {stim }}$ and $\boldsymbol{\sigma}_{\text {conf. }}$ For each subject, we fit 3 data features - the average psychometric function, the difference between psychometric functions under high and low confidence [P(right_hi) - P(right_lo)] and the RT-confidence relationship. Choice probabilities were binned into 5 quantiles; RT was z-scored and binned into 7 quantiles. The normalised discrepancy function between model and data was calculated as follows:

$$
E=\sum_{i, j} \frac{\left|e_{i, j}-m_{i, j}\right|}{n_{i} N_{i}}
$$

where $E$ is the total discrepancy for a particular parameter setting, $e$ and $m$ are experimental and model values, respectively, $i$ indexes each function, and $j$ indexes each point on the ith function. $n_{i}$ is the number of points in the ith curve, and $N_{i}$ is its range of values. This denominator ensures the contribution of each curve to the total discrepancy is expressed as a fraction between 0 and 1 . Fits were carried out using exhaustive gridsearch across a 2dimensional parameter surface. Error surfaces were inspected to avoid local minima. Bestfitting parameters from each individual subject are included in the Table below. We observed 
that $E$ was less sensitive to changes in $\boldsymbol{\sigma}_{\text {conf }}$ than $\boldsymbol{\sigma}_{\text {stim. }}$. The fit for one subject did not result in a clearly-defined global minimum, and is thus excluded.

Supplementary Table 3 - BOLD activation

\begin{tabular}{|c|c|c|c|c|}
\hline Contrast & Region & MNI $[x, y, z]$ & $\overline{\mathrm{K}_{\mathrm{E}}}$ & $P$ - value \\
\hline \multirow[t]{3}{*}{$\begin{array}{l}\text { Difference in } \\
\text { value (increase) }\end{array}$} & vmPFC & {$[12,56,4]$} & 190 & $p<0.005^{*}$ \\
\hline & Precuneus & {$[-6,-52,16]$} & 444 & $p<0.0001^{*}$ \\
\hline & Left STS & {$[-45,-67,-25]$} & 136 & $p<0.05^{*}$ \\
\hline $\begin{array}{l}\text { Difference in } \\
\text { value (decrease) }\end{array}$ & $\mathrm{ACC}$ & {$[9,20,49]$} & 184 & $p<0.005^{*}$ \\
\hline \multirow[t]{2}{*}{$\begin{array}{l}\text { Confidence } \\
\text { (increase) }\end{array}$} & vmPFC & {$[12,47,-11]$} & 190 & $p<0.005^{*}$ \\
\hline & Precuneus & {$[-3,-46,34]$} & 113 & $p<0.05^{*}$ \\
\hline \multirow[t]{5}{*}{$\begin{array}{l}\text { Confidence } \\
\text { (decrease) }\end{array}$} & $\mathrm{ACC}$ & {$[9,17,40]$} & 198 & $p<0.0001^{*}$ \\
\hline & $\begin{array}{l}\text { Left anterior } \\
\text { Insula }\end{array}$ & {$[-48,11,-2]$} & 233 & $p<0.0001^{*}$ \\
\hline & $\begin{array}{l}\text { Right anterior } \\
\text { Insula }\end{array}$ & {$[51,8,4]$} & 135 & $p<0.0001^{*}$ \\
\hline & $\begin{array}{l}\text { Left subthalamic } \\
\text { nucleus (STN) }\end{array}$ & {$[-6,-10,7]$} & 34 & $p<0.005^{*}$ \\
\hline & Right RLPFC & {$[27,47,28]$} & 42 & $p<0.005^{\S}$ \\
\hline
\end{tabular}

$\mathrm{K}_{\mathrm{E}}=$ cluster size $;{ }^{*}=\mathrm{FWE}$ cluster corrected $; \S=$ small volume corrected (SVC) within 8-mm sphere centred on the coordinates $[36,44,28]$ taken from [Fleming et al 2012] 
Supplementary Table 4 - List of items

\begin{tabular}{|l|}
\hline Item name \\
\hline Kettle Chips (Sweet Chilli) \\
\hline Wotsits Crisps \\
\hline Twix Bar \\
\hline M\&Ms \\
\hline Lion Bar \\
\hline Bounty \\
\hline Walkers Cheese \& Onion Crisps \\
\hline Milky Way Bar \\
\hline Brunch Bar \\
\hline Cadburys Twirl \\
\hline Monster Munch Crisps \\
\hline Skittles \\
\hline Nestle Milky Bar \\
\hline Nestle Kit Kat \\
\hline Mars Bar \\
\hline Dairy Milk Turkish Delight \\
\hline Cadbury Crunchie \\
\hline KP Salted Peanuts \\
\hline Doritos Cool Original Crisps \\
\hline
\end{tabular}

